\title{
KHOAN VO-KHAC
}

\section{La régularisation dans les problèmes combinatoires et son application au problème de sectorisation}

Revue française d'automatique, d'informatique et de recherche opérationnelle. Recherche opérationnelle, tome 5, n V2 (1971), p. 59-77.

<http://www.numdam.org/item?id=RO_1971_5_2_59_0>

(C) AFCET, 1971, tous droits réservés.

L'accès aux archives de la revue « Revue française d'automatique, d'informatique et de recherche opérationnelle. Recherche opérationnelle » implique l'accord avec les conditions générales d'utilisation (http://www.numdam.org/ legal.php). Toute utilisation commerciale ou impression systématique est constitutive d'une infraction pénale. Toute copie ou impression de ce fichier doit contenir la présente mention de copyright.

\section{Numdam}

Article numérisé dans le cadre du programme

Numérisation de documents anciens mathématiques

http://www.numdam.org/ 
R.I.R.O.

(5e année, V-2, 1971, p. 59-77)

\title{
LA REGULARISATION \\ DANS LES PROBLEIMES GOIMBINATOIRES \\ ET SON APPLIGATION \\ AU PROBLEME DE SEGTORISATION
}

\author{
par Khoan Vo-KHAC
}

Résumé. - Dans les problèmes combinatoires, les évaluations nécessaires pour l'exploitation par ramification, guidage et contrôle se font, en général, à l'aide d'un classement hiérarchique basé sur certaines notions topologiques. Nous nous proposons de chercher la topologie la plus adaptée dans le cas du problème de sectorisation. Deux exemples numériques sont traités en détail.

Le problème de tournées a été fort longtemps l'objet de nombreuses études mathématiques. Avec Dantzig et Ramser [3] comme pionniers, diverses méthodes heuristiques (mais simples) ou rigoureuses (mais impraticables) ont été données [1] [2] [6] [7]. Certaines de ces méthodes sont moyennement bonnes, d'autres franchement mauvaises. Ces méthodes ont toutes en commun la notion de voisinage, c'est-à-dire l'ensemble des points voisins d'un point donné. Il est erronné de croire que deux clients sont voisins lorsque le coût de passage entre eux est faible, comme nous l'avons montré dans une précédente note [8]. Dans cette même note, nous avons appliqué notre idée au problème des circuits hamiltoniens. Dans la présente note, nous allons l'appliquer au problème de sectorisation.

\section{LE PROBLEME DE SECTORISATION}

\section{$1^{\circ}$ Données et notations du problème}

On se donne un graphe $G$ à $N+1$ sommets $\omega_{0}, \omega_{1}, \ldots, \omega_{N}$; chaque sommet du graphe représente une localité (ville, commune ou zone). Le graphe est valorisé par une matrice $c(i, j)$; le nombre $c(i, j)$ représente le coût pour aller 
du sommet $i$ au sommet $j$. On supposera que la matrice $c(i, j)$ est " pleine » et symétrique (footnote); mais on ne supposera pas que le graphe est euclidien.

Parmi tous ces sommets, il en existe un plus particulier que les autres qu'on appellera le centre $0 \mathrm{du}$ graphe (ou le Dépôt distributeur); les autres sommets seront appellées les points de livraison (ou les clients).

On appelle multicycle (centré) hamiltonien tout cycle passant une fois et une seule par chaque client et au moins une fois par le dépôt distributeur. Si le multicycle se compose exactement de $J$ boucles, alors on l'appelle un $J$-cycle. Le coût $C$ d'un multicycle donné, est par définition, la somme des coûts formant le multicycle.

\section{$2^{\circ}$ Position du problème (sans contraintes)}

a) On cherche, parmi l'ensemble des multicycles hamiltoniens, un multicycle optimal (i.e. le moins coûteux).

\section{Autrement dit :}

On cherche un nombre $J$ et une partition de l'ensemble $\Omega=\left\{\omega_{1}, \ldots, \omega_{N}\right\}$ en $J$ classes disjointes $\Omega_{1} \ldots, \Omega_{j}$ telles que : $\sum_{j=1}^{J} C_{j}=\min$ (où $C_{j}$ désigne le coût du cycle hamiltonien optimal de l'ensemble $\Omega_{j} \cup 0$ ). Chaque classe $\Omega_{j}$ s'appalle alors un secteur. Dans la plupart des cas, le nombre $J$ est donné à l'avance (ou calculable).

b) On peut se contenter de faire d'abord la partition de $\Omega$ en secteurs (on cherchera ensuite dans chaque secteur le cycle hamiltonien optimal par la méthode R.C.G. exposée dans [8]. Un tel problème sera appelé problème de sectorisation (sans contraintes).

REMARQUE. - Il y a une analogie entre le problème de sectorisation et le problème de stratification aux moindres carrés [9] : dans le premier problème c'est le cycle hamiltonien optimal de chaque secteur qui intervient; dans le second problème, c'est le cycle hamiltonien moyen (évalué en distances quadratiques) de chaque secteur qui intervient (en effet, on peut montrer facilement que la dispersion interne d'un «segment » n'est autre que le coût moyen (calculé en distances quadratiques) des cycles hamiltoniens passant par tous les points du segment.

Footnote : Dans l'appendice, on donne un algorithme rendant pleine une matrice partiellement vide; d'autre part, la méthode que nous allons exposer s'applique, avec des modifications évidentes aux matrices non symétriques. 


\section{$3^{\circ}$ Contraintes}

Les contraintes sont diverses et sont spécifiques à chaque problème. Ici, nous nous tiendrons à une seule classe de contraintes assez répandue : les contraintes de capacité (par exemple contraintes de tonnage ou contraintes d'effectifs).

A chaque client $\omega$, on associe un vecteur à plusieurs dimensions $\vec{V}(\omega)$ appelé capacité de $\omega$. La capacité d'un secteur $\Omega_{j}$ est, par définition

$$
\vec{V}\left(\Omega_{j}\right)=\sum_{\omega \in \Omega_{j}} \vec{V}(\omega)
$$

On impose alors aux secteurs $\Omega_{j}$ les contraintes suivantes :

$$
\vec{m}_{j} \leqslant \vec{V}\left(\Omega_{j}\right) \leqslant \vec{M}_{j}
$$

Dans le problème des tournées le plus simple, l'espace des contraintes est unidimensionnel; par ailleurs $M_{j}$ est donné (capacité des camions), mais $m_{j}$ est calculable; il se peut aussi que $m_{j}=M_{j}$.

\section{TRANSFORMATIONS ADMISSIBLES}

\section{$1^{\circ}$ Définition}

On appelle transformation admissible toute transformation, sur la matrice des coûts, laissant inchangée la hiérarchie relative des multi-cycles hamiltoniens.

En particulier, une transformation admissible laisse inchangé l'ensemble des solutions optimales.

Il est trivialement vrai, que la composition de deux transformations admissibles est une transformation admissible.

\section{$2^{\circ}$ Exemples}

Soient $\lambda$ un nombre réel et $\left(\alpha_{i}\right)$ un vecteur à $N+1$ dimensions $(i=0, \ldots, N)$

On appelle homothétie $(\lambda)$ la transformation consistant à multiplier la matrice des coûts par la constante $\lambda$.

On appelle translation $\left(\alpha_{i}\right)$ la transformation consistant à ajouter à chaque rangée $i$ (ligne $e$ t colonne) la constante $\alpha_{i}$; dans le cas particulier où $\alpha_{i}=0$ sauf $i=i_{0}$, la transformation sera appelée translation partielle du sommet $i_{0}$; dans le cas où tous les $\alpha_{i}$ sont égaux, la transformation s'appelle une équi-translation.

$n^{\circ}$ V-2, 1971. 


\section{Proposition}

(a) Toute homothétie $(\lambda)$ est admissible si $\lambda>0$;

(b) Toute translation partielle $\left(\alpha_{i}\right)$ d'un client $i(i \neq 0)$ est admissible

$\mathrm{Si}$, de plus le nombre $J$ des secteurs est imposé à l'avance, alors

(c) Toute translation partielle $\left(\alpha_{0}\right)$ du dépôt distributeur est admissible

(d) Toute équitranslation $(\alpha)$ est admissible).

En effet, dans le cas $a$, le coût total est multiplié par $\lambda$; dans le cas $b$, le coût total s'augmente de $2 \alpha_{i}$; dans le cas $c$, le coût total s'augmente de $2 J \alpha_{0}$ dans le cas $d$, le coût total s'augmente de $2(N+J) \alpha$.

\section{PONDERATION DES COUTS}

\section{$1^{\circ}$ Matrice des coûts admissibles}

On appelle matrice de coûts admissibles l'image de la matrice des coûts bruts par une transformation admissible.

On appelle liste hiérarchique des arêtes le classement des arêtes selon l'ordre croissant des coûts.

L'intérêt de ces notions est immédiat : on peut toujours remplacer la matrice des coûts par une matrice de coûts admissibles pour résoudre le problème de sectorisation; cependant, la liste hiérarchique des arêtes change en général. Or, il existe une infinité de matrices de coûts admissibles. Nous allons voir, cependant, que deux d'entre eux sont plus importants que les autres et qu'une combinaison linéaire judicieusement choisie de ces deux coûts permet d'obtenir un coût admissible meilleur que tous les autres.

\section{$2^{\circ}$ Coûts centralisés et coûts régularisés}

On appelle coût centralisé entre deux clients $i$ et $j$ le coût $c_{0}(i, j)$ défini par :

$$
c_{0}(i, j)=c(i, j)-c(i, o)-c(o, j)
$$

On appelle coût régularisé entre deux clients $i$ et $j$ le coût $c_{\infty}(i, j)$ défini par :

$$
c_{\infty}(i, j)=c(i, j)-\frac{\Gamma(i)+\Gamma(j)}{N-2}+\frac{\Gamma}{(N-1)(N-2)}
$$

où

$$
\Gamma(i)=\sum_{\substack{k \neq 0 \\ k \neq i}} c(i, k) \quad \text { et où } \quad \Gamma=\sum_{i \neq 0} \Gamma(i)
$$

Revue Française d'Informatique et de Recherche opérationnelle 
Il est évident que la matrice des coûts centralisés et la matrice des coûts régularisés sont des matrices de coûts admissibles (dans le cas de la centralisation, cela revient à la composition des translations partielles $\alpha_{i}=-c(i, o)$ avec $i \neq o$ ); dans le cas de la régularisation, cela revient à la composition des translations partielles

$$
\alpha_{i}=\left(\frac{\Gamma}{2(N-1)(N-2)}-\frac{\Gamma(i)}{N-2} i \neq 0\right)
$$

Intuitivement, la centralisation tient compte de la position relative d'une arête $(i, j)$ par rapport au dépôt distributeur, alors que la régularisation tient compte de la position relative de l'arête $(i, j)$ par rapport aux autres clients. Faut-il utiliser les coûts centralisés ou les coûts régularisés? L'interprétation économique suivante permet d'en dégager la réponse.

\section{$3^{\circ}$ Interprétation économique}

Considérons deux clients $i$ et $j$, situés sur deux secteurs différents. Nous allons calculer le gain moyen obtenu en reliant $i$ et $j$.

Désignons par $i^{\prime}$ et $j^{\prime}$ les sommets reliés à $i$ et $j$ dans la solution initiale, et reliés entre eux dans la solution modifiée.

On doit distinguer trois cas :

a) Cas où $i^{\prime}$ et $j^{\prime}$ sont des clients (fig. 1).

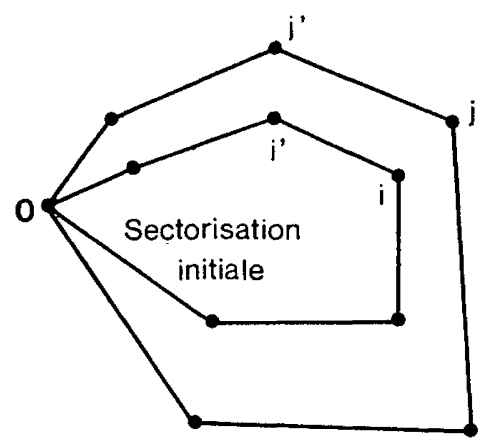

Figure 1

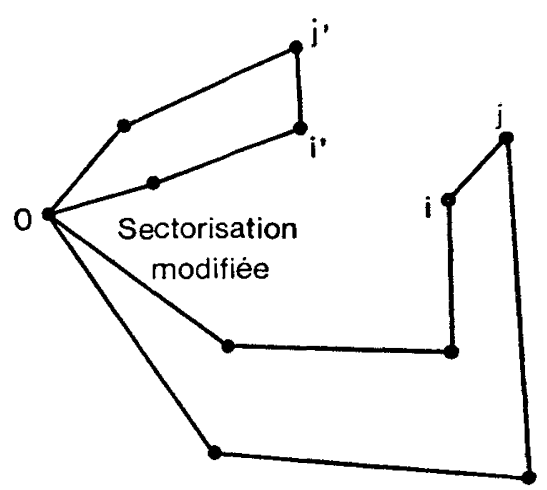

Ce gain est égal à la valeur moyenne de :

$$
c\left(i, i^{\prime}\right)+c\left(j, j^{\prime}\right)-c(i, j)-c\left(i^{\prime}, j^{\prime}\right)
$$

La valeur moyenne de $c\left(i, i^{\prime}\right)$ est $: \frac{1}{N-2} \sum_{\substack{i^{\prime} \neq i \\ i^{\prime} \neq j \\ i^{\prime} \neq 0}} c\left(i, i^{\prime}\right)=\frac{\Gamma(i)-c(i, j)}{N-2}$ $n^{\circ}$ V-2, 1971. 
La valeur moyenne de $c\left(j, j^{\prime}\right)$ est $: \frac{\Gamma(j)-c(i, j)}{N-2}$

La valeur moyenne de $c\left(i^{\prime}, j^{\prime}\right)$ est :

$$
\frac{1}{(N-2)(N-3)} \sum_{\substack{i^{\prime} \neq i, j, 0 \\ j^{\prime} \neq i, j, 0}} c\left(i^{\prime}, j^{\prime}\right)=\frac{\Gamma-2 \Gamma(i)-2 \Gamma(j)-2 c(i, j)}{(N-2)(N-3)}
$$

Le gain moyen est donc, par linéarité :

$$
\begin{aligned}
\frac{\Gamma(i)+\Gamma(j)}{N-2}(1+ & \left.\frac{2}{N-3}\right)-\frac{\Gamma}{(N-2)(N-3)} \\
& \quad-c(i, j)\left(1+\frac{2}{N-2}+\frac{2}{(N-2)(N-3)}\right)=-\frac{N-1}{N-3} c_{\infty}(i, j) .
\end{aligned}
$$

Dans ce cas, le gain moyen obtenu est donc proportionnel à l'opposé du coût régularisé

b) Cas où $i^{\prime}$ et $j^{\prime}$ coïncident avec le dépôt distributeur (fig. 2).

Dans ce cas, au lieu de 2 secteurs, on obtient un seul secteur.
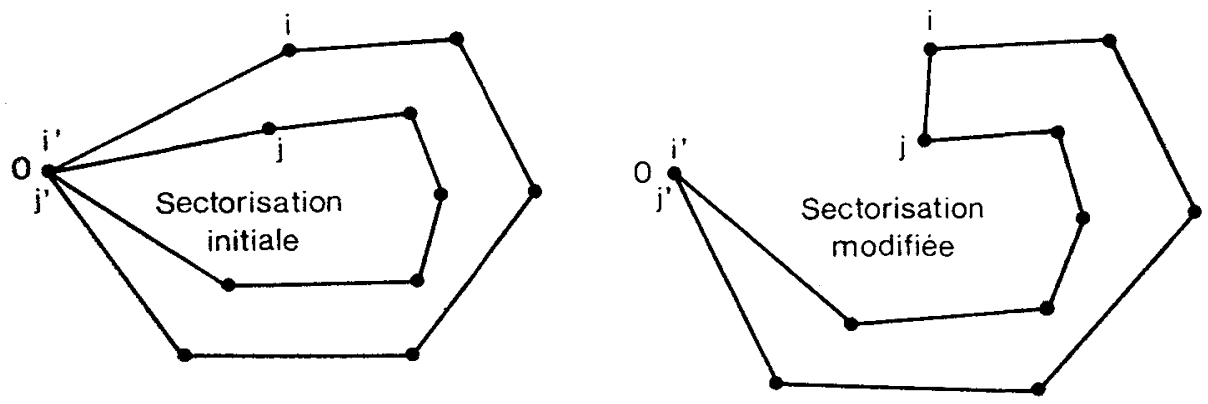

Figure 2

Le gain est alors égal à :

$$
c(i, 0)+c(0, j)-c(i, j)
$$

Autrement dit, il est égal à l'opposée du coût centralisé. (fig. 3).

c) Cas où $i^{\prime}$ est le dépôt distributeur et $j^{\prime}$ un client (ou réciproquement) 

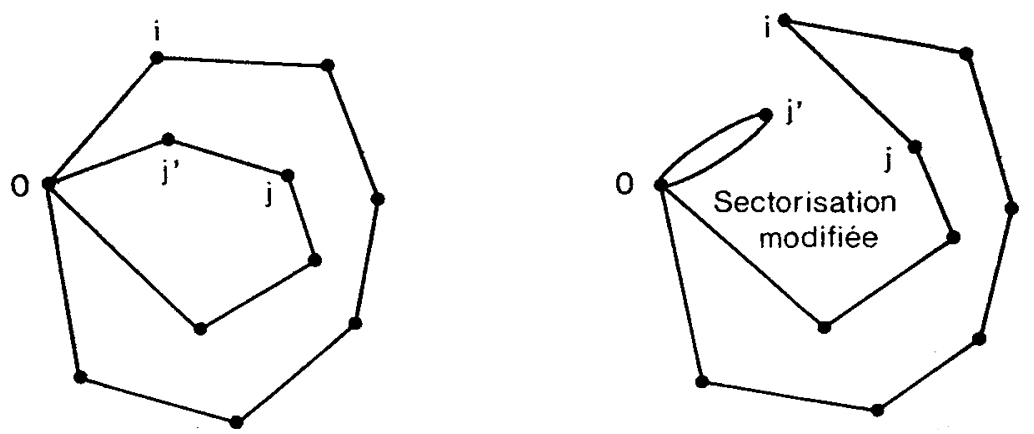

Figure 3

Un calcul facile montre que le gain moyen obtenu est égal à

$$
\begin{gathered}
-\frac{N c_{0}(i, j)+(N-2) c_{\infty}(i, j)+2 \Gamma(0)}{2(N-2)} \\
*^{*}
\end{gathered}
$$

On peut donc conclure que le coût admissible le plus approprié au problème des tournées doit être une combinaison linéaire des coûts régularisés et centralisés de la forme $\mu_{\infty} c_{\infty}(i, j)+\mu_{0} c_{0}(i, j)$. Nous allons montrer, par la méthode des centres fictifs, que les coefficients de cette combinaison linéaire est, pour $J$ fixé, donné par :

$$
\mu_{\infty}=N-2 \text { et } \mu_{0}=J
$$

$4^{\circ}$ Méthodes des centres fictifs (fig. 4)

Considérons $J$ centres fictifs $0_{1}, \ldots, 0_{J}$ possédant les propriétés suivantes :

$$
\begin{aligned}
& d\left(0_{\alpha}, \quad i\right)=d(0, i) \text { pour tout } i=1,2, \ldots, N \\
& d\left(0_{\alpha}, 0_{\beta}\right)=\Lambda \quad \text { pour tout } \alpha, \beta=1,2, \ldots, J
\end{aligned}
$$
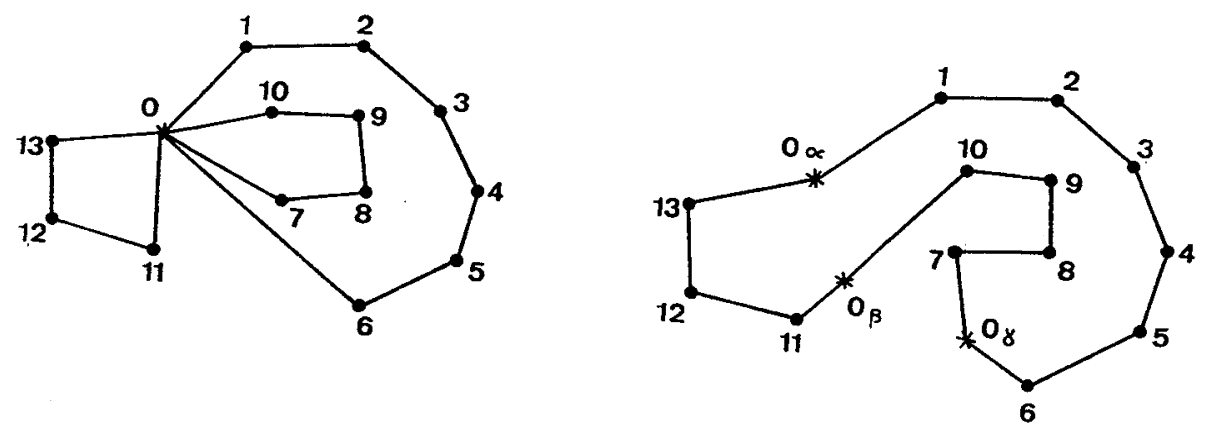

Figure 4

$n^{\circ}$ V-2, 1971. 
où $\Lambda$ est une constante. Pourvu que $\Lambda$ soit assez grand, on peut considérer la $J$ - sectorisation (sans contraintes) comme un problème de commis-voyageur à $(N+J)$ sommets.

Posons :

$$
\begin{aligned}
& \Gamma^{\neq}(i)=\Gamma(i)+J c(i, 0) \\
& \Gamma^{\neq}(0)=\Gamma(0)+(J-1) \Lambda \quad \text { où } \quad \Gamma(0)=\sum_{i=1}^{N} c(i, 0) \\
& \Gamma^{\neq}=\Gamma+J \Gamma(0)
\end{aligned}
$$

Les coûts régularisés pour ce problème de commis-voyageur à $(N+J)$ sommets sont alors [8] donnés par :

$$
c_{\infty}^{\neq}(i, j)=c(i, j)+\frac{\Gamma^{\neq}}{(N+J-1)(N+J-2)}-\frac{\Gamma^{\neq}(i)+\Gamma^{\neq}(j)}{N+J-2}
$$

A un facteur additif près, on a donc :

$$
c_{\infty}^{\neq}(i, j)=\frac{(N-2) c_{\infty}(i, j)+J c_{0}(i, j)}{N+J-2}
$$

\section{Conclusion}

Le meilleur coût admissible dans la J-sectorisation est le coût pondéré $c_{\infty}^{\neq}(i, j)$.

\section{$5^{\circ}$ Utilisations pratiques}

a) Dans la pratique, on substitue aux coûts pondérés (qui sont négatifs en général) par les affinités pondérées qui leurs sont proportionnelles.

Ces affinités pondérées sont définies par :

$$
\begin{aligned}
a^{\neq}(i, j) & =\Gamma(i)+\Gamma(j)+J[c(0, i)+c(0, j)]-(N+J-2) c(i, j) \\
\text { si } i \quad \text { et } j \neq 0 & \\
a^{\neq}(0, i) & =\Gamma(i)-(N-2) c(0, i) .
\end{aligned}
$$

b) L'obtention de la matrice des affinités pondérées à partir de la matrice des coûts bruts se réalise, en fait, très simplement :

on multiplie la matrice des coûts par $-(N+J-2)$ puis on ajoute à chaque rangée $i(\neq 0)$ la constante $\Gamma(i)+J c(0, i)$.

On n'ajoute rien à la rangée 0 .

c) Cette matrice des affinités pondérées va remplacer la matrice des coûts bruts. (Il n'est pas nécessaire de stocker cette dernière matrice dans la mémoire 
de l'ordinateur.) En effet, soit $C$ le coût d'un multicycle hamiltonien quelconque et $A^{*}$ l'affinité pondérée du même cycle. On a alors :

$$
A^{\neq}+(N+J-2) C=2(\Gamma+J \Gamma(0)),
$$

ce qui permet de trouver $C$ connaissant $A^{\neq}$.

d) Les affinités pondérées permettent de dresser la liste hiérarchique des arêtes. Cette liste sera constamment utilisée dans la suite.

\section{METHODES DE SECTORISATION}

Si l'on désire avoir rapidement une bonne solution, on utilisera la sectorisation hiérarchique. Si l'on désire pousser le calcul jusqu'à une solution optimale, on utilisera la méthode R.G.C.

\section{$1^{\circ}$ Sectorisation hiérarchique}

Nous rappelons les contraintes de capacité :

$$
\vec{m}_{j} \leqslant \vec{V}\left(\Omega_{j}\right) \leqslant \vec{M}_{j} \quad(j=1,2, \ldots, J)
$$

où les vecteurs sont multidimensionnels.

Nous supposerons, pour simplifier le raisonnement, que ces vecteurs sont unidimensionnels (une seule sorte de contrainte de capacité). Dans la plupart des cas, les $M_{j}$ sont donnés, mais les $M_{j}$ doivent être calculés à chaque étape. A vrai dire, on ne sait pas quel est le secteur $\Omega_{1}$, ce n'est pas forcément le premier secteur formé. On suppose donc que l'on est dans la situation suivante : quelques secteurs sont formés, et quelques autres restent à former. On pose :

$$
\begin{aligned}
& m=\inf m_{j} \text { (inf à prendre sur les } j \text { restant) } \\
& M=\sup M_{j} \text { (sup à prendre sur les } j \text { restant) } \\
& \mu=M-\inf V(\Omega j) \text { (inf à prendre sur les } j \text { restants). }
\end{aligned}
$$

Un secteur est dit admissible si sa capacité est entre $m$ et $M$ ( $m$ sera appelé le seuil d'admissibilité).

Un secteur admissible est dit saturé si sa capacité est supérieure à $\mu$ ( $\mu$ sera appelé le seuil de saturation).

L'algorithme qu'on va décrire est, en quelque sorte, agglomératif et récurrentiel.

\section{Étape première}

a) On commence par former le premier secteur admissible. Pour cela, on prend comme " noyau » le client en tête du classement hiérarchique. Ce noyau va grossir en capturant d'autres clients. Un client (ou un noyau) est capturé $n^{\circ}$ V-2, 1971. 
par un autre noyau si leur affinité de mélange est la plus grande possible, et s'il n'y a pas dépassement de capacité. En première approximation, on peut admettre que l'affinité de mélange entre deux noyaux $\mathcal{H}$ et $\mathcal{N}$ est égale à $\sup \left\{a^{\neq}(m, n) ; m \in \mathcal{M}, n \in \mathcal{N}\right\}$

(On note qu'un client peut être capturé par un autre client $i$ même si $i$ est déjà relié aux deux autres clients ; en effet, il s'agit, pour le moment, de former des secteurs.)

On continue jusqu'à ce que le premier secteur soit admissible.

b) Une fois que le premier secteur est admissible, la liste hiérarchique indiquera que l'on peut commencer à former un deuxième secteur, ou l'on doit continuer à grossir le premier secteur jusqu'à ce qu'il soit saturé.

c) Quand un secteur est saturé, on enlève ses clients de la matrice des affinités (pondérées) et on calcule à nouveau $m, M$, et $\mu$.

\section{Deuxième étape}

S'il existe déjà un deuxième noyau formé, alors on continue à le grossir jusqu'à le rendre admissible, puis saturé.

Sinon, on commence par former ce deuxième noyau en prenant le premier client (restant) intervenant en tête du classement hiérarchique.

\section{Étape générale}

Et ainsi de suite...

\section{Arrêt}

On termine la sectorisation lorsqu'il n'y a plus possibilité d'agglomérer des secteurs sans dépassement de capacité.

\section{Formation des circuits à l'intérieur de chaque secteur}

Dans chaque secteur, on applique alors les méthodes du problème du commis-voyageur (cf. [8]) pour trouver un circuit hamiltonien optimal.

\section{Contrôle de la qualité de la solution}

a) Pour contrôler la qualité de la solution obtenue, on cherche une majoration (évaluation par excès).

On appelle affinité d'un sommet la demi-somme des deux affinités les plus grandes des arêtes issues de ce sommet.

Une majoration s'obtient en prenant la somme des affinités de tous les clients et $\boldsymbol{J}$ fois l'affinité du dépôt distributeur. 
b) Pour améliorer cette évaluation par excès, on ajoute au préalable à la rangée 0 de la matrice des affinités (pondérées) la constante

$$
\alpha_{0}=\Gamma(0)+(J-1)\left(\frac{2(N-1) \Gamma(0)-\Gamma}{N(N-1)}\right)
$$

on calcule la majoration, puis on retranche du nombre obtenu le nombre $2 J \alpha_{0}$. On remarque que $-2(N-1) \Gamma(0)+\Gamma$ représente la moyenne des coûts centralisés de toutes les arêtes.

c) Quelle que soit les transformations utilisées, on note cependant qu'un tel contrôle n'est pas toujours excellent, car il ne tient pas compte des contraintes de capacité. Par conséquent, même si l'on a obtenu par la sectorisation hiérarchique la solution optimale, ce contrôle pourrait laisser croire que l'on soit encore loin de l'optimalité.

Autrement dit : la sectorisation hiérarchique fournit en général une solution nettement meilleure que ne l'indique le contrôle.

\section{$2^{\circ}$ Méthode de ramification, guidage et contrôle}

Le principe général de la méthode R.G.C. (exploitation par ramification, guidage et contrôle) a été exposé dans Vo-Khac [8].

\section{a) Ramification}

Ici, on fait une biramification : introduire un nouveau client ou ne pas l'introduire dans le secteur à former. Ce nouveau client s'introduit hiérarchiquement (en utilisant la liste hiérarchique) en respectant les contraintes de capacité. D'autre part, un secteur doit toujours être admissible avant la formation d'un autre secteur, conformément aux indications précédentes.

\section{b) Guidage}

L'évaluation par défaut (guidage) s'obtient en faisant, à chaque sommet de l'arborescence, une sectorisation hiérarchique comme précédemment. Cependant, pour l'évaluation des coûts des circuits dans chaque secteur, on peut se contenter d'utiliser les affinités pondérées (sans utilisation de la méthode R.G.C.).

\section{c) Contrôle}

L'évaluation par excès (contrôle) se fait comme précédemment. On note aussi que :

(i) Lorsqu'on interdit l'appartenance d'un client $i$ à un secteur, on interdit toutes les arêtes reliant ce client $i$ aux clients du secteur.

(ii) Dans un secteur partiellement formé et presque saturé, on peut introduire les contraintes de capacité dans l'évaluation par excès (l'exemple qui suit permet de comprendre comment on introduit ces contraintes). 


\section{REMARQUE}

Dans le contrôle comme dans le guidage, on peut admettre qu'une $J$-sectorisation optimale est toujours meilleure que $(J+1)$-sectorisation optimale, ce qui est vrai dans la plupart des cas, mais pas toujours.

\section{EXEMPLES D'APPLICATION}

\section{$1^{\circ}$ Premier exemple}

Il s'agit d'un problème à un dépôt distributeur et à 8 clients, trouvé dans Marconi [6].

a) Données. - La capacité des camions est uniforme et égale à 20000 . Ci-dessous les quantités à livrer et la matrice des coûts (partie triangulaire inférieure).

\begin{tabular}{|c|c||c|c|c|c|c|c|c|c|c|}
\hline $\begin{array}{c}\text { QUANTITÉ } \\
\text { A LIVRER }\end{array}$ & & $\begin{array}{c}\text { DÉPOT } \\
0\end{array}$ & 1 & 2 & 3 & 4 & 5 & 6 & 7 & 8 \\
\hline 40000 & 0 & & 23 & 49 & 24 & 21 & -24 & 84 & 32 & 71 \\
\hline 6000 & 1 & 18 & & 72 & 215 & 260 & 215 & 163 & 135 & 86 \\
\hline 4000 & 2 & 07 & 25 & & 161 & 126 & 193 & 133 & 201 & 208 \\
\hline 4000 & 3 & 10 & 07 & 06 & & 149 & 208 & 108 & 184 & 119 \\
\hline 6000 & 4 & 22 & 13 & 22 & 19 & & 173 & 265 & 85 & 92 \\
\hline 5000 & 5 & 20 & 13 & 06 & 04 & 20 & & 60 & 208 & 183 \\
\hline 4000 & 6 & 10 & 21 & 17 & 20 & 12 & 30 & & 124 & 171 \\
\hline 7000 & 7 & 11 & 19 & 03 & 05 & 29 & 06 & 20 & & 191 \\
\hline 4000 & 8 & 16 & 35 & 12 & 23 & 38 & 19 & 24 & 16 & \\
\hline
\end{tabular}


Comme le total des quantités à livrer est 40000 et comme la capacité d'un camion est 20000 , on va d'abord essayer de faire une bi-sectorisation; la capacité de chaque secteur doit donc être exactement 20000 ; ici on a donc : $m_{j}=M_{j}=m=M=20000$ pour tout $j=1,2$. Un secteur est admissible et saturé si sa capacité est 20000 ; on va donc former un secteur jusqu'à ce que sa capacité atteigne 20000 ; les clients restant constituent alors le deuxième secteur.

b) Calculs préliminaires.

$$
\Gamma_{0}=114, \quad \Gamma=964, \quad \alpha_{0} \simeq 126
$$

La matrice des affinités pondérées se trouve dans la partie triangulaire supérieure du tableau précédent.

\section{c) Application de l'algorithme hiérarchique.}

Le client 4 forme le noyau du premier secteur; ce noyau capture le client 6 , puis le noyau $\{4,6\}$ capture le client 1 , finalement le noyau $\{4,6,1\}$ capture le client 3 ; le secteur $\{1,3,4,6\}$ est alors saturé. Le deuxième secteur $\{2,5,7,8\}$ est constitué des clients restants.

Il nous reste à former les circuits optimaux dans chaque secteur; la méthode R.G.C. exposé dans [8] donne immédiatement le circuit 064130 pour le premier secteur et les circuits 082570,087520 et 085720 pour le deuxième secteur.

L'affinité pondérée du bicycle obtenu est 1560 ; le coût du bicycle est donc : $\frac{2(964+2 \times 112)-1560}{8}=103$.

Quelle est la qualité de la solution obtenue? Nous pouvons prendre pour évaluation par excès le nombre $265+60+215+\frac{1}{2} 215+210+208$

$$
+208+\frac{1}{2} 208+201+197+\frac{1}{2} 191+\frac{1}{2} 175+\frac{1}{2} 158-4 \times 126=1733,5
$$

La solution obtenue est donc à $\frac{1733-1560}{1733} \simeq 10 \%$ au plus de la solution optimale; nous verrons cependant que ce contrôle est trop pessimiste.

d) Algorithme R.G.C.

Le résultat peut se résumer dans les arborescences suivantes (fig. 5 et 6).

Le guidage (fig. 5) montre qu'il faut prendre comme premier secteur les clients $4,6,1,3$. Les autres clients forment alors le second secteur. A l'intérieur $n^{\circ} \mathrm{V}-2,1971$. 
de chaque secteur, on forme alors le circuit optimal; finalement on trouve

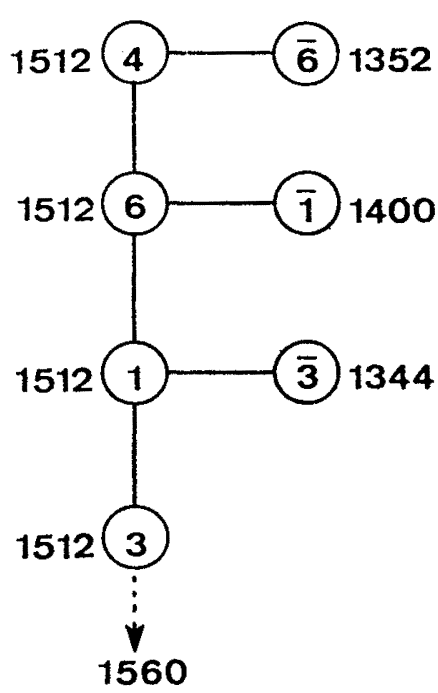

Figure 5 que le bicycle possède l'affinité 1560 .

Le contrôle se fait en mettant en évidence deux sortes de contraintes de capacités : contrainte de tonnages $\left(t_{1}=t_{1}=20000\right)$ et contrainte d'effectifs $\left(n_{1}=n_{2}=4\right)$. La valeur $-\infty$ indique que 1 'on ne peut faire une bisectorisation en suivant la branche indiquée. Exploitant 17 sommets, on montre que la solution obtenue est une bissectorisation optimale (fig. 6).

\section{$2^{\circ}$ Deuxième exemple}

Il s'agit d'un problème à un dépôt distributeur et à 12 clients, posé par Dantzig et Ramser [3], repris par Clark et Wright [2], puis par Bertier [1].

a) Données

Ci-dessous, la matrice des coûts (partie triangulaire inférieure) et les quantités à livrer.

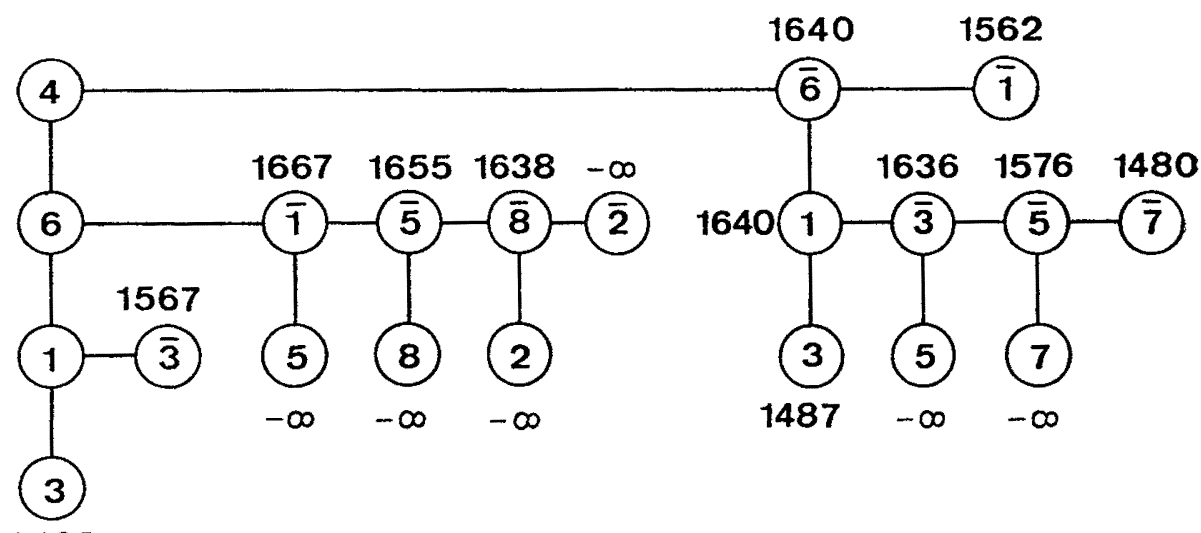

Figure 6

Il existe un nombre illimité de camions de capacité 4000 , trois camions de capacité 5000 , quatre camions de capacité 6000 .

b) Calcul préliminaire.

Comme le total des quantités à livrer est 18200 , on va d'abord essayer de faire une quadri-sectorisation. On voit alors que $M_{j}=M=6000$ pour 


\begin{tabular}{|c|c|c|c|c|c|c|c|c|c|c|c|c|c|}
\hline $\mathcal{I}$ & \begin{tabular}{c}
\multirow{N}{*}{} \\
1
\end{tabular} & $\stackrel{2}{\stackrel{2}{2}}$ & ర్ల & $m_{m}^{\infty}$ & $\stackrel{\infty}{+}$ & $\mid \begin{array}{l}\infty \\
\infty\end{array}$ & $\mathcal{F}$ & 点 & 誌 & 勇 & 总 & $\underset{\infty}{\mathbb{N}}$ & \\
\hline$=$ & $\underset{1}{\mathbb{N}}$ & $\stackrel{+}{\stackrel{ \pm}{5}}$ & ઠ్సి & ల্লি & 弚 & $\underset{\sim}{\mathbb{\infty}}$ & 导 & $\tilde{\tilde{n}}$ & 영 & $\frac{9}{7}$ & $\approx$ & & 음 \\
\hline 으 & 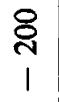 & $\stackrel{\infty}{=}$ & స్ & $\stackrel{\widetilde{m}}{\tilde{m}}$ & : & \& & 莳 & $\frac{0}{n}$ & 范 & ஜ్లి & & $\infty$ & 으 \\
\hline$a$ & $\underset{1}{\cong}$ & 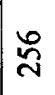 & $\stackrel{\infty}{d}$ & $\underset{\infty}{\sim}$ & $\stackrel{ \pm}{\sim}$ & $\tilde{\sigma}$ & F & $\frac{\Delta}{n}$ & $\underset{0}{0}$ & & $\approx$ & $\approx$ & ণ \\
\hline$\infty$ & $\frac{J}{1}$ & শ্ন & న్ & స్ & $\stackrel{\infty}{\underset{d}{\sim}}$ & 过 & గి & 京 & & 10 & 0 & \pm & 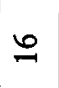 \\
\hline$r$ & $\underset{1}{\stackrel{\overbrace{}}{1}}$ & $\stackrel{\infty}{\sim}$ & ర్ల & $\stackrel{\infty}{\sim}$ & ஜ̊ & $\mid$\begin{tabular}{l}
$\infty$ \\
\multirow{\infty}{*}{}
\end{tabular} & 苦 & & 오 & $\mathscr{0}$ & 으 & $\stackrel{\infty}{\sim}$ & 요 \\
\hline 0 & 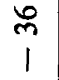 & ల్ల & 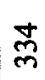 & $\underset{N}{N}$ & ষ্লি & 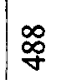 & & r & $\Rightarrow$ & 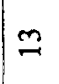 & $\approx$ & $\approx$ & $\hat{\sim}$ \\
\hline$n$ & $\begin{array}{c}\infty \\
1\end{array}$ & 六 & $\stackrel{\infty}{\forall}$ & $\underset{ల}{ \pm}$ & $\cong$ & & $a$ & 으 & 10 & $\tilde{N}$ & ㄱ. & $\stackrel{\infty}{\sim}$ & లి \\
\hline$\forall$ & in & $\stackrel{\ddot{m}}{\mathrm{~g}}$ & $\stackrel{\mathscr{o}}{+}$ & $\frac{1}{6}$ & & 2 & $\stackrel{\infty}{\sim}$ & $\approx$ & $\check{m}$ & $\vec{\gamma}$ & సे & $\bar{m}$ & নి \\
\hline$m$ & $\Phi$ & 点 & $\widetilde{\widetilde{\delta}}$ & & 으 & $\bar{N}$ & ৪ & $\hat{N}$ & m & $\mathscr{q}$ & $\bar{m}$ & m & gे \\
\hline$\sim$ & I & రి & & $1-$ & $=$ & $\underline{1}$ & $\tilde{\lambda}$ & ㄴ. & 요 & i & 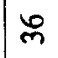 & 守 & fo \\
\hline- & $\stackrel{\infty}{\tilde{n}}$ & & $n$ & $\approx$ & $\mathbb{N}$ & $\bar{N}$ & $\stackrel{\sim}{N}$ & $\bar{m}$ & $\approx$ & ñ & $F$ & g & $\vec{n}$ \\
\hline \multirow[t]{2}{*}{0} & & a & \pm & $\bar{\sim}$ & $\ddot{\sim}$ & $\approx$ & $\approx$ & ల్ల & | & $\mid \infty ্$ & $\mathscr{F}$ & in & $\tilde{n}$ \\
\hline & 0 & - & $N$ & $m$ & $\theta$ & $\ln$ & 6 & 12 & $\infty$ & a & 10 & $\Rightarrow$ & $\simeq$ \\
\hline 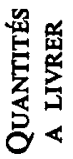 & 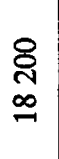 & ষ্ণ & $\stackrel{8}{2}$ & $\begin{array}{l}8 \\
n \\
-1\end{array}$ & 字 & 옹 & ষ্ণ & $\stackrel{\text { I }}{2}$ & \&̊̊ & $\stackrel{8}{\infty}$ & 8 & 8 & $\stackrel{8}{\Xi}$ \\
\hline
\end{tabular}


$j=1,2,3,4$. Les nombres $m_{j}$ dépendent de l'étape; au départ, on a : $m$ inf $m_{j}=18200-3 \times 6000=200$. Quant aux centrations, on trouve : $\Gamma(0) \stackrel{j}{=} 364, \Gamma=3088$ (d'où $\alpha_{0} \simeq 476$ ). La matrice des affinités pondérées se trouve dans la partie supérieure du tableau précédent.

\section{c) Algorithme hiérarchique.}

On trouve aisément les 4 secteurs suivants : $\{12,11,10,7\},\{8,9,6\},\{2,1$, $3,4\},\{5\}$. La formation des circuits à l'intérieur de chaque secteur se fait par la méthode R.C.G. décrite dans [8]. Dans le troisième secteur, on trouve un circuit optimal unique 0-1-2-3-4-0; dans le deuxième, on trouve deux circuits optimaux 0-6-8-9-0 et 0-6-9-8-0; dans le premier on trouve quatre circuits optimaux à savoir $0-7-10-11-12-0,0-7-10-12-11-0 ; 0-7-11-12-10-0$ et $0-7-12$ 11-10-0.

L'affinité pondérée du quadricycle obtenue est 5028 . Son coût est donc $C=\frac{2(3088+4 \times 364)-5028}{14}=290$.

Pour contrôler la qualité de la solution obtenue, on applique la méthode indiquée précédemment en ajoutant à chaque affinité des arêtes passant par 0 le nombre $\alpha_{0}=476$, en calculant la majoration puis en retranchant de cette majoration $8 \alpha_{0}$. On trouve comme évaluation par excès le nombre 6487 . La solution trouvée est donc à $\frac{6487-5028}{6487} \simeq 20 \%$ au plus de la solution optimale. Malgré ce résultat assez pessimiste, la solution trouvée est probablement optimale.

d) Algorithme R.G.C.

Le résultat du guidage est reproduit ci-dessous (fig. 7)

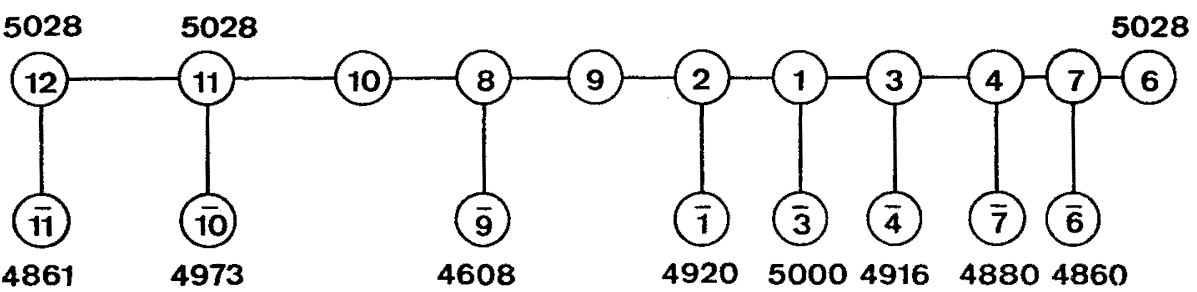

Figure 7 


\section{CONCLUSION GENERALE}

Les méthodes préconisées (algorithme hiérarchique et méthode R.G.C.) ont été appliquées à un grand nombre de problèmes de sectorisation. Les conclusions dégagées sont les suivantes :

\section{$1^{\circ}$ Comparaison entre les coûts pondérés et les coûts centralisés}

Les coûts bruts sont proscrits. Deux coûts admissibles et possédant des significations économiques intéressantes sont les coûts pondérés et les coûts centralisés.

L'utilisation des coûts bruts a la tendance à répartir les points de livraison en zone trop " compactes".

L'utilisation des coûts centralisés a la tendance à réunir les points de livraison «en circonférence » autour du dépôt distributeur; on commence en général à grouper les points éloignés du dépôt distributeur (même si ces points sont assez éloignés entre eux); les coûts centralisés ne considèrent que la position des points de livraison par rapport au centre et ne considèrent pas leur position relative. L'utilisation des coûts pondérés permet d'obtenir une sectorisation ni trop compacte, ni trop éloignée. Ces coûts tiennent compte de la position relative de tous les clients et aussi de celle du dépôt distributeur.

Lorsque le rapport $\frac{N}{J}$ est supérieur à 5 , les coûts centralisés donnent de mauvais résultats. Lorsque ce rapport est petit, ils donnent sensiblement les mêmes résultats que les coûts pondérés.

\section{$2^{\circ}$ Valeur de la sectorisation hiérarchique}

La sectorisation hiérarchique (utilisant les coûts pondérés) est extrêmement simple et donne de bons résultats. Si l'on se contente d'une solution très rapide, on peut l'utiliser. Mais la méthode d'exploitation par ramification et guidage est meilleure.

\section{$3^{\circ}$ Valeur et possibilité d'utilisation de la méthode R.G.C.}

La ramification et le guidage sont simples et donnent de très bons résultats.

Ils donnent, par une simple «descente » une bonne solution réalisable. On peut toujours, si l'on veut trouver de meilleures solutions, remonter l'arborescence et se ramifier dans la branche où l'on trouve un guidage voisin de la solution trouvée.

Le contrôle est long à faire, et le calcul est pénible. Il devient inutilisable sur des problèmes de grandes tailles. 


\section{$4^{\circ}$ Établissement de la matrice des coûts et des vecteurs de contraintes de capacités}

Il nécessite la connaissance du temps de déchargement et de rechargement au dépôt, du temps de livraison (par client et par tonne déchargée), des vitesses moyennes dans diverses zones (en rase campagne, dans les districtes urbains ou en plein centre ville). Il ne faut pas non plus oublier de distinguer la vitesse d'approche de la vitesse de ramassage. Il est aussi prudent d'ajouter un temps mort supplémentaire pour l'ensemble de la tournée.

\section{APPENDICE}

\section{$1^{\circ}$ Le problème : plus courte distance dans un réseau}

Étant donné un graphe quelconque à $I$ sommets, valorisé par une matrice des coûts $c_{0}(i, j)$, trouver un chemin joignant deux sommets $i$ et $j$ de coût minimum.

Il existe plusieurs algorithmes : celui de Dantzig [4] est le plus connu; cependant celui de $\mathrm{Hu}$ [5] est le plus performant. Nous décrivons ici, en quelques lignes, la méthode de $\mathrm{Hu}$, renvoyant les lecteurs à l'article de $\mathrm{Hu}$ pour les astuces et les détails complémentaires.

\section{$2^{\circ}$ Algorithme de $\mathbf{H u}$}

On pose :

$$
c_{k}(i, j)=\min \left\{c_{k-1}(i, j), c_{k-1}(i, k)+c_{k-1}(k, j)\right\}
$$

Le nombre $c_{k}(i, j)$ n'est autre que le coût de la route le moins coûteux qui relie $i$ et $j$ en passant par les sommets dont le numéro d'identification est $\leqslant k$.

Alors $c_{I}(i, j)$ est le coût du chemin minimum cherché.

L'ordinogramme est très simple. L'entrée $c(i, j)$ est remplacé par la somme $c(i, k)+c(k, j)$ si cette somme est plus faible, et reste inchangée si cette somme est plus grande ou égale (et cela pour $k=1,2, \ldots, I$ ).

D'après $\mathrm{Hu}, 1$ 'algorithme est très performant. Ploquin ingénieur à la CEGOS (utilisant l'algorithme pour les besoins d'un software de typologie pour D18) confirme ce jugement.

Remerciement : l'article a vu le jour après de fructueuses discussions avec les ingénieurs de la CEGOS. 


\section{BIBLIOGRAPHIE}

[1] Bertier (P.), «Procédures pour élaborer des tournées de distribution », METRA, série spéciale, no 8,1966 (114 pages).

[2] Clarke (G.) et Wright (J. W.), "Scheduling of vehicles from a central depot to a number of delivery points », JORSA, (1964), $\mathrm{n}^{\circ} 4,568-581$.

[3] Dantzig (G. B.) et Ramser (J. H.), " The truck dispatching problem », Management Science 6 (1959), 80-91. Optimum Routing of Gasoline Delivery Trucks. Proc. Fifth World Petrleum Cong., Sect VIII (1959).

[4] Dantzig (G. B.), "On the Shortest Route through a Network ", Management Science 6 (1960), $\mathrm{n}^{\circ} 8$.

[5] Hu (T. C.), "A decomposition algorithm for shortest paths in a network », JORSA, 16 (1968), 91-102.

[6] MARCoNi (R.), «La Ricerca operativa applicata al problema dei rifornimente ", QUADERNI R.O., $\mathrm{n}^{\circ} 1$ (1962).

[7] Tompa (M.) (en hongrois), «Organisation du service des véhicules par des méthodes mathématiques », ELELMEZESI IPER. MAGYAR 21 (1967), n 10, 314-316.

[8] Vo-Khac (Khoan), «La régularisation dans les problèmes combinatoires », R.I.R.O., 3e année (1969), $\mathrm{n}^{\circ}$ V-I, 91-104.

[9] Vo-Khac (Khoan) et NGHiem (Phong Tuan), « Typologie et segmentation, la méthode de stratification aux moindres carrés ", Hommes et Techniques, mai 1968, 425-436. 